\title{
A FRAMEWORK FOR ROUTING IN LARGE AD-HOC NETWORKS WITH IRREGULAR TOPOLOGIES
}

\author{
Marc Heissenbüttel, Torsten Braun, David Jörg, Thomas Huber* \\ Institute of Computer Science and Applied Mathematics \\ University of Bern, Switzerland \\ \{heissen, braun, joerg, thuber\} @iam.unibe.ch
}

\begin{abstract}
In this paper, we consider routing in large wireless multihop networks with possibly irregular topologies. Existing position-based routing protocols have deficiencies in such scenarios as they always forward packets directly towards the destination. Greedy routing frequently fails and costly recovery mechanisms have to be applied. We propose the Ants-based Mobile Routing Architecture (AMRA) for optimized routing, which combines position-based routing, topology abstraction, and swarm intelligence. AMRA routes packets along paths with high connectivity and short delays by memorizing past traffic and by using ant-like packets to discover shorter paths. The geographic topology abstraction allows AMRA to cope with high mobility and large networks. Simulative evaluation indicate that compared to other position-based routing AMRA finds significantly shorter paths with only marginal overhead protocols.
\end{abstract}

Keywords: Ad-hoc networks, routing, swarm intelligence

\section{Introduction}

Routing in wireless multihop networks has generated a lot of interest and a large number of routing protocols have been proposed. In positionbased routing protocols like GFG [1] and GPSR [2], nodes are aware of their positions e.g. through GPS. Each node forwards packets greedily to one of its neighbors closer to the destination. A recovery mechanism has to be applied if no neighbor is closer and this greedy routing fails.

*The work presented in this paper was supported (in part) by the National Competence Center in Research on Mobile Information and Communication Systems (NCCR-MICS), a center supported by the Swiss National Science Foundation under grant number 5005-67322. 
Position-based protocols require only little control traffic and do not need to maintain paths. Thus, they are scalable and robust to changes in the network, which make them the preferred choice for large and highly dynamic networks. However, position-based routing protocols show also some shortcomings.

- Routing a packet along the line-of-sight between the source and destination may often not be possible in realistic networks due unpopulated areas, mountains, or lakes. Thus, greedy routing of position-based protocols will fail and the recovery mechanism must be applied. The path chosen may be very suboptimal.

- Each packet is sent completely independently of all others. If greedy routing fails and the recovery mechanism forwards the packet along a very long path even though a much shorter exists, all subsequent packets will follow the longer path. The protocols have no way to adapt and to learn from experiences.

To summarize, the stateless approach of position-based protocols is not only the reason for their advantages, but is also the source of new drawbacks such as the lack of knowledge about network topology on a large scale. Thus, if we assume that the overall node distribution in the network remains quite static and only varies slowly over time, it is beneficial to accumulate such information at the nodes to facilitate communication with distant nodes.

We propose the Ants-based Mobile Routing Architecture (AMRA) whose objective is to overcome these aforementioned drawbacks of conventional position-based protocols. It is designed for routing in large wireless multihop networks with possibly tens of thousands of nodes with irregular topologies. In such scenarios, AMRA is able to find more optimal paths than other position-based protocols by memorizing past traffic such that packets are not routed necessarily directly towards the destination anymore. The required memory to keep track of the traffic can be kept small, in the order of some hundred bytes, by applying an aggregated and fisheye-like view on the network. Furthermore, if only few data traffic is in the network and existing paths are not known, additional ant-like control packets can be emitted to actively discover shorter paths. Both types of packets, data and ants, increase the probability for their traveled path depending on the encountered quality. Thus, packets are attracted to travel along the good path already traveled by other packets, which in turn increase the probability for these paths even more.

This principle of self-reinforcing of traveled paths through packets is basically the principle of ant-colony optimization where ants find shortest paths between the nest and a food source. The ant colony optimiza- 
tion principle has been applied lately to routing in ad-hoc networks in several papers $[3,5,4]$. All these ant-based routing algorithms are similar to other topology-based protocols and have a route discovery, a route maintenance, and a route error phase. They mainly make use of the ant colony optimization to improve the resilience and reliability of paths or to improve existing paths compared to other topology-based protocols. Therefore, they still have the same characteristics of other topologybased protocols such as large control traffic overhead and, thus, are not suited for large networks with highly dynamic topologies as considered in this paper.

The remainder of this paper is organized as follows. In Section 1, we describe AMRA and the used protocols in detail. AMRA is evaluated in Section 2 by simulations and finally Section 3 concludes the paper.

\section{The Ants-based Mobile Routing Architecture}

AMRA is a two-layered framework with three independent protocols rather than an actual routing protocol. Three specific protocols are presented exemplarily within the AMRA framework. The two protocols used on the upper layer are called Topology Abstracting Protocol (TAP) and Mobile Ants-Based Routing (MABR). Straight Packet Forwarding (StPF) is situated on the lower layer and functions as an interface to the physical network for MABR. Due to lack of space only the general concepts are given in this section, for more details cf. [6].

\section{Topology Abstraction Protocol (TAP)}

TAP is the key to make routing scalable and provides in a transparent manner an aggregated and static topology with fixed "logical routers" (LR) and fixed "logical links" (LL) to MABR. Logical routers are fixed geographical areas of equal size arranged in a grid to cover the whole global area. Depending on its current position, each node is part of one specific logical router. A node can easily detect, based on its position, when it crosses the border of the current logical router and then it automatically becomes a member of the new logical router. In order to scale to large networks, each logical router groups other logical routers into zones $Z_{i, j}$ as shown in Fig. 1 . The zone size increases exponentially with the distance $i$ to the center router and allows covering large areas with few zones. This is justified by the circumstance that from the view of a fixed node, close-by nodes that move some distance may be located in an entirely different direction, whereas the same movement of a node far away only marginally affects the direction. It is important to notice that 
the view of zones is relative. Each logical router resides in the center of its own zone model.

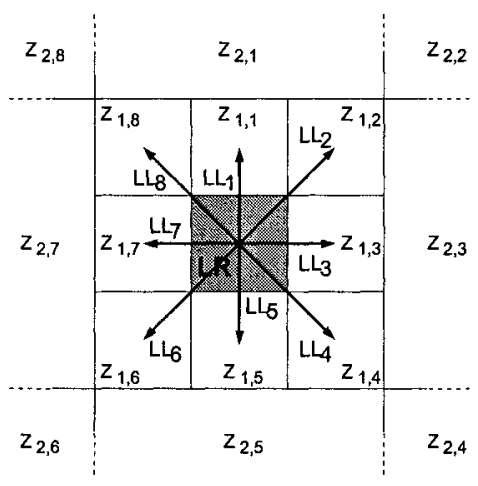

Figure 1. Logical router LR in the center, the zones in its view $Z_{i, j}$, and its logical links $L L_{k}$ indicated as arrows

A logical link $L L_{k}$ represents a path along a straight line to an adjacent logical router over possibly multiple physical hops. In this way, we introduce a static logical topology on the network independent of the actual node distribution.

\section{The Mobile Ants-Based Routing Protocol (MABR)}

The routing protocol MABR operates on top of abstract topology provided by TAP and thus does not have to cope with changing topologies. Basically, all what MABR has to do now is that whenever a node receives or overhears a packet, it determines where the packet originates from and from which direction it arrived. More precisely, it determines the source zone $Z_{i, j}$ of the packet by the coordinates of the source node as given in the packet header. Note that this zone is relative to the view of the current node. Furthermore, the node determines the last logical router in which the packet was forwarded before having entered the current logical router, i.e., it determines the logical link $L L_{k}$ which approximates most closely the followed path over the last few physical hops. Nodes maintain a probabilistic routing table where all the zones and the logical links are organized in rows and columns, respectively. The value of the field in the routing table corresponding to the determined zone $Z_{i, j}$ and logical link $L L_{k}$ is increased. The other seven entries in the row of $Z_{i, j}$ are decreased proportionally such that the sum over all logical links in a row for a certain zone remains 1. A high value indicates that there exists a path in the direction of that logical link to the respective zone. 
Eventually, the best paths will emerge and MABR is able to circumvent areas with bad or no connectivity. Then, data packets will always be routed over logical links with high connectivity such that greedy routing is possible. MABR routes data packets by determining to which zone a packet should be routed from the destination coordinates as given in the packet header. The node then selects the logical link with the highest probability to this zone. Consequently, data packets are routed logicalhop by logical-hop over the logical links, i.e. from one logical router to one of its adjacent logical routers and so on. Furthermore, ants can be transmitted periodically to explore new paths if there is only little data traffic. Unlike data packets, ants are routed purely position-based, i.e., they are not influenced by the probabilistic routing of MABR but forwarded directly towards the destination by StPF. If a node does not have a logical link with a high probability, the data packets are also routed purely position-based and adapt therefore the role of ants.

In irregular topology, the logical link pointing directly towards the destination zone may often not have a high value as no packets arrive out of this direction. Consider again the same exemplarily topology as before in Fig. 2. A node $S$ that wants to route to a destination node $D$ does not forward the packet towards node $C$, because $L L_{3}$ pointing in this direction has a very low probability as no packets from zone $Z_{3,3}$ traveled over this link. $S$ forwards the packet either over $L L_{1}$ or $L L_{6}$ because the received data packets originating from zone $Z_{3,3}$ arrived from the direction of $L L_{1}$ and $L L_{3}$. The possible paths for packets from a node $D$ in zone $Z_{3,3}$ to $S$ are depicted exemplarily. Thus, for any destination node located in zone $Z_{3,3}$, the packets are also routed over these two links with high probability.

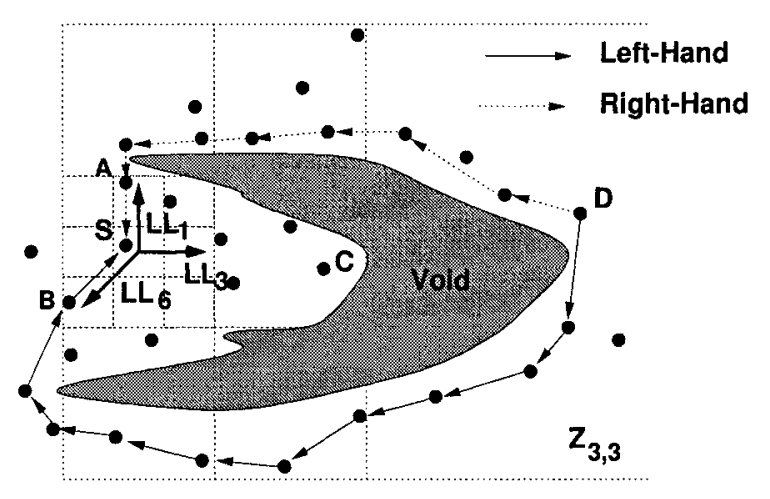

Figure 2. Routing packets to $Z_{3,3}$ over $L L_{1}$ and $L L_{6}$ 
If a node moves to another logical router, its view on the network changes, and it adapts its routing table accordingly by having the probability of all logical links approaching a uniform distribution. The reason is that previously collected information about good paths looses its relevance because zones and links are relative to a node's view and do no longer correspond to previous geographical areas.

\section{Straight Packet Forwarding (StPF)}

$\mathrm{StPF}$ is a position-based routing protocol and responsible to physically forward packets over the logical link determined by MABR to the next logical router. StPF can be basically any standard position-based routing protocol such as GFG/GPSR $[1,2]$. In GFG/GPSR, packets are forwarded to the neighbor closest to the final destination. If no such neighbor exists and greedy routing fails, GFG/GPSR applies a perimeter routing mode to recover. Therefore, each node extracts locally a planar subgraph of the actual network graph, which is necessary to avoid loops, and forwards packets on the faces of this subgraph according to the right-hand rule. Packets are again routed in greedy mode as soon as they are received at a node that is closer to the final destination than where the packet entered the perimeter mode.

\section{Evaluation}

As AMRA is designed for large networks, we also conducted simulations with several thousand nodes. Unfortunately, realistic network simulators like ns-2 are not able to run such large simulations. Therefore, we implemented and simulated AMRA in a Java network simulator and compared it to GFG/GPSR and a shortest path algorithm. The Java simulator does not account for any physical propagation medium properties or MAC layer functionality and thus is able to run simulations with thousands of nodes. Therefore, packets cannot be dropped due to collisions or congestion and packets do not experience delay. We use the hop count metric in order to asses the performance. The hop count metric is typically considered a good indication for the delay because CSMA based MAC protocols such as IEEE 802.11 have high cost for acquiring the medium. The nominal transmission range and the logical router side length were both set to $250 \mathrm{~m}$. The results are averaged over 10 simulation runs and given with a double-sided $90 \%$ confidence interval. Data packets are transmitted periodically between two randomly chosen communication peers at a rate of 1 packet/s. The simulation time was set to $1800 \mathrm{~s}$, but no data is transmitted in the initial first $900 \mathrm{~s}$ to reach a stable state of the mobility model. AMRA was always simulated 
with unidirectional and bidirectional traffic between the source and the destination. The reason is that AMRA can use traffic flowing in the opposite direction to update the routing tables towards the destination. On the other hand, GFG/GPSR and the shortest path algorithm are not affected by bidirectional traffic and thus they were only simulated with unidirectional traffic.

To simulate large networks with irregular topologies, we use the restricted random waypoint mobility model [7]. The model defines rectangular city areas and highways connecting selected cities, but otherwise is similar to the standard random waypoint mobility model. Nodes choose a next waypoint within their current city or in one of the adjacent cities connected by highways. Consequently, there may be void areas with no nodes such that direct routing between some cities is not possible. A typical scenario is depicted in Fig. 3 with four cities and three highways.

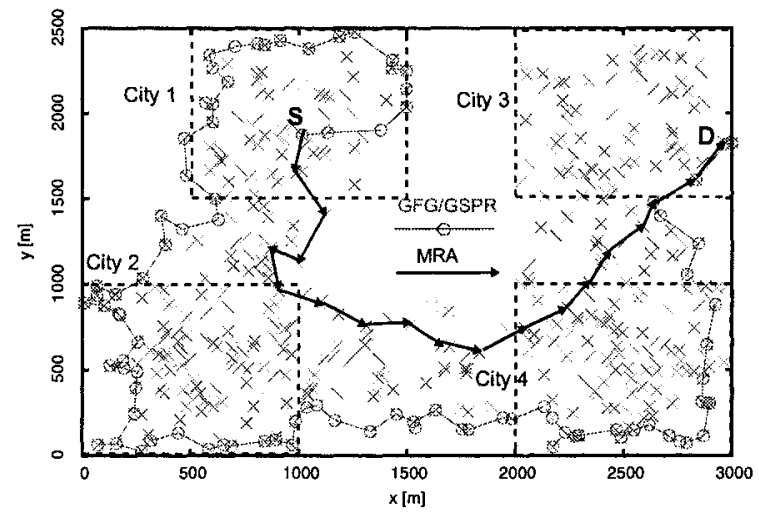

Figure 3. Path of MRA and GFG/GPSR in irregular topology

We defined four cities of $1000 \mathrm{~m} \times 1000 \mathrm{~m}$ interconnected by three highways with 500 nodes on an area of $3000 \mathrm{~m} \times 2500 \mathrm{~m}$. Nodes in the city move at a speed in the interval $[1,15] \mathrm{m} / \mathrm{s}$ and at a higher speed on the highway $[10,30] \mathrm{m} / \mathrm{s}$. A typical path chosen by AMRA and GFG/GPSR is also shown in the figure. Although it is definitely a worst-case scenario for GFG/GPSR, it again clearly highlights the problem of position-based protocols, namely the inability to know which are good paths to a distant node on a large scale. We first conducted simulations where the number of transmitted ants was varied and we had a fixed number of traffic sources set to 10 .

In Fig. 4, we can see that GFG/GPSR has on average an about 2.5 times higher hop count than the shortest possible path. Considering 
the fact that often the traffic flow is between nodes in the same city or one of the adjacent cities, we may conclude that the hop count for traffic flows between non-adjacent cities is much more than 2.5 times the shortest path. If nodes are in adjacent cities, routing along a straight line between them is possible and the performance of GFG/GPSR is almost identical to the shortest path.

AMRA with only unidirectional traffic and no ants performs even worse than GFG/GPSR. However, as soon as few ants are transmitted the hop count drops sharply. With only 50 ants transmitted per second in the whole network, i.e. with 500 nodes, each node transmits an ant each 10 seconds, the hop count is about 15 compared to 10 of the shortest path and 25 for GFG/GPSR. The further increase of ants does not further reduce the hop count however. On the other hand, if we have bidirectional traffic, the hop count is completely independent of the number of ants. The data packets in the opposite direction are sufficient to establish high probability entries in the routing tables.

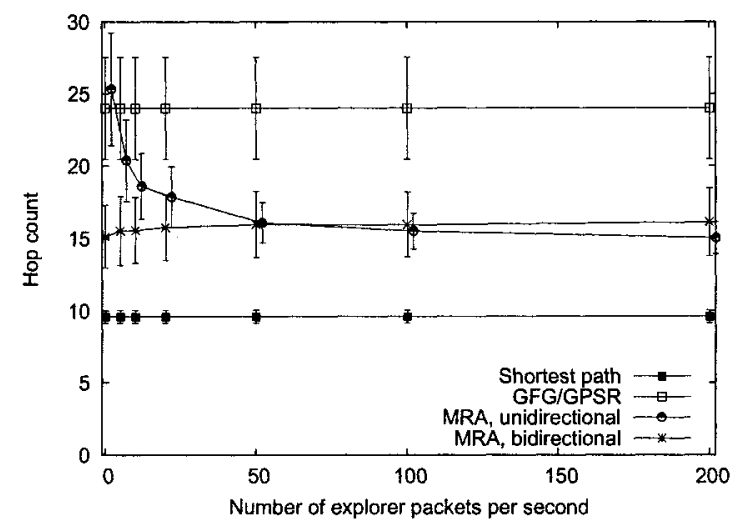

Figure 4. Irregular network with varying number of ants

In a next step, we simulated a scenario where no ants are transmitted at all and only the number of traffic flows was varied Fig. 5. Again, the performance of GFG/GPSR shows an about 2.5 times higher hop count than the shortest path. Unlike before, the graphs for GFG/GPSR and the shortest path are no longer exactly constant, but only statistically constant within the confidence intervals. The reason is that, unlike the number of ants, a varying number of sources may yield slightly different results among the different simulation runs. AMRA with bidirectional traffic remains almost unaffected by the number of traffic flows, i.e. traffic flowing in different directions does not distort the entries in 
the routing tables for traffic flows to other destinations. As before where we had 10 traffic flows, AMRA with unidirectional traffic suffers if we have no ants and only few traffic flows. The chance that a node has overheard a lot of traffic to a given destination zone is low and, thus, when it has to forward a packet to that zone the risk is high that it forwards the packet in a wrong direction. However, as more traffic flows there are in the network, the performance of AMRA with unidirectional traffic approaches the performance of AMRA with bidirectional traffic. If we have sufficient traffic, the entries in the routing tables are updated accurately by the data packet themselves. The reason is that if there are no useful entries in the routing tables, data packets are routed purely position-based and thus adopt the role of ants.

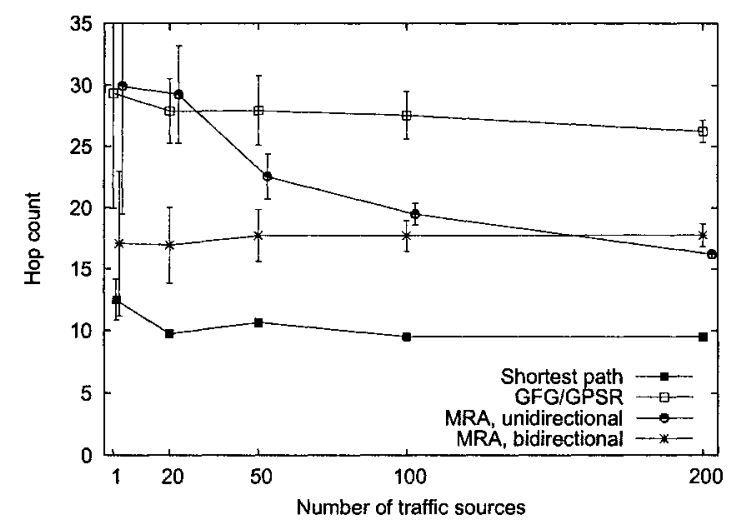

Figure 5. Irregular network with varying data traffic and no ants

\section{Conclusion}

In this paper, we presented the Ants-based Mobile Routing Architecture (AMRA), which makes use of topology abstraction, a principle from swarm intelligence, and position-based routing. AMRA is used to optimize routing in large network with irregular topologies where routing along a a straight line towards the destination is not possible. Results showed that AMRA is able to cope efficiently with irregular network topologies, i.e. realistic topologies for large networks. In a scenario with a horseshoe-like topology, AMRA was able to find paths that are up to $40 \%$ shorter than of GFG/GPSR. Consequently, AMRA would also yield much shorter delays and reduce congestion in the network. In simple and flat network topologies, AMRA performed comparable to GFG/GPRS. Unlike GFG/GPSR, AMRA uses ants to discover new paths and, thus, 
introduces additional control traffic however. Simulation showed that the number of ants can be kept small. For scenarios with bidirectional traffic or a lot of unidirectional traffic, even no ants are required. Therefore, the overhead compared to GFG/GPSR reduces to little additional memory to store the routing table. Realistic network traffic is typically bidirectional, e.g. simply because TCP is used on the transport layer. We can summarize the main features of AMRA as follows.

- AMRA allows nodes to learn by memorizing past traffic such that disadvantageous paths are avoided and packets are routed along paths with high connectivity.

- Due to the abstract topology, AMRA can easily cope with high mobility and is scalable in terms of number of nodes and the covered geographical area of the network.

- The overhead due to ants can be minimized as only few or even none are required to find good paths.

\section{References}

[1] P. Bose, P. Morin, I. Stojmenovic, and J. Urrutia, "Routing with guaranteed delivery in ad hoc wireless networks," in Proceedings of ACM Workshop on Discrete Algorithms and Methods for Mobile Computing and Communications (DIALM '99), Seattle, USA, Aug. 1999.

[2] B. Karp and H. T. Kung, "GPSR: Greedy perimeter stateless routing for wireless networks," in Proceedings of the 6th Annual ACM/IEEE International Conference on Mobile Computing and Networking (MOBICOM '00), Boston, USA, Aug. 2000, pp. 243-254.

[3] M. Günes, M. Kähmer, and I. Bouazizi, "Ant-routing-algorithm (ARA) for mobile multi-hop ad-hoc networks - new features and results," in Proceedings of the 2nd Mediterranean Workshop on Ad-Hoc Networks (Med-Hoc-Net'2003), Mahdia, Tunesia, June 2003.

[4] M. Roth and S. Wicker, "Termite: Ad-hoc networking with stigmergy," in Proceedings of IEEE Global Telecommunications Conference (GLOBECOM'03), San Francisco, USA, Dec. 2003.

[5] G. Di Caro, F. Ducatelle, and L. Gambardella, "AntHocNet: An adaptive nature-inspired algorithm for routing in mobile ad hoc networks," European Transactions on Telecommunications, vol. 16, no. 2, 2005.

[6] M. Heissenbüttel, "Routing and Broadcasting in Ad-Hoc Networks", Ph.D. dissertation, University of Bern, Switzerland, June 2005.

[7] L. Blazevic, S. Giordano, and J. Y. L. Boudec, "Self organized terminode routing simulation," in Proceedings of MSWiM 2001, Rome, Italy, July 2001. 